\title{
Desafíos contemporáneos en el desarrollo del sector eléctrico colombiano
}

\author{
Contemporary challenges in developmentof the Colombian electricity sector
}

Recibido: 11-02-2019 • Aprobado: 23-05-2019 • Página inicial: 87 - Página final: 111

\author{
Freddy León Paime*
}

\begin{abstract}
Resumen: el sector eléctrico es vital en el desarrollo de la sociedad colombiana. El presente documento analiza cuáles son los desafíos claves que está enfrentando este conjunto de organizaciones, a partir de una revisión a los datos globales que hacen parte del desempeño del sector eléctrico colombiano. Fruto de tal ejercicio, se identifica la presencia de seis fenómenos que pueden suponer un ambiente problemático tanto para el sector, como para la sociedad. Estos tienen que ver con la concentración de la propiedad de los activos del sistema, el lento crecimiento de la capacidad instalada, la difícil incorporación de las tecnologías limpias, la conformación de una estructura del mercado que impide la aparición de nuevos actores, la ausencia de una estrategia clara frente al cambio climático, y la conformación de estructuras de rendición de cuentas centradas en el inversor del sector institucional.
\end{abstract}

Palabras clave: comportamiento organizacional, índices de desempeño, sector eléctrico.

\begin{abstract}
The electricity sector is vital in the Development of Colombian society. He This document analyzes what are the key challenges that this is facing set of organizations, from a review of the global data that are part of the performance of the Colombian electricity sector. Fruit of such exercise, the presence is identified of six phenomenathatmay involveaproblematic environment for both the sector, As for society. These have to do with the concentration of ownership of system assets, the slow growth of the installed capacity, the difficult incorporation of clean technologies, shaping a market structure that prevents the appearance of new actors, the absence of a clear strategy against climate change, and the conformation of accountability structures focused on the institutional sector investor
\end{abstract}

Keywords: Organizational behavior, performance indices, electricity sector.

JEL: Q01

\footnotetext{
* Profesor Investigador de la Universidad Libre. Contador público de la Universidad Nacional de Colombia, Magister en Investigación social y Doctor en Educación de la Universidad Distrital Francisco José de Caldas. edisonf.leonp@utadeo.edu.co-leonpaime7@gmail.com ORCID: https://orcid.org/0000-0002-7897-1945
} 


\section{Contexto Loon-aime, E}

\section{Desafios contemporâneos no desenvolvimento do setor elétrico colombiano}

Resumo: o setor elétrico é vital no Desenvolvimento da sociedade colombiana. Ele Este documento analisa quais são as principais desafios que isso enfrenta conjunto de organizações, de um revisão dos dados globais que fazem parte do desempenho do setor elétrico colombiano. Fruto de tal exercício, a presença é identificada de seis fenômenos que podem envolver uma ambiente problemático para o setor, Quanto à sociedade. Isso tem a ver com a concentração de propriedade de ativos do sistema, o lento crescimento da capacidade instalada, a difícil incorporação de tecnologias limpas, moldando estrutura de mercado que impede o aparecimento de novos atores, a ausência de uma estratégia clara contra as mudanças climáticas ea conformação de estruturas de prestação de contas focadas em o investidor do setor institucional.

Palavras-chave: comportamento organizacional, índices de desempenho, setor elétrico. 


\section{Introducción}

El desarrollo del sector eléctrico ha jugado un papel importante como pieza angular sobre la que se construye el proceso de modernización en Colombia, pues ha logrado una expansión significativa en las últimas décadas, con lo que se ha asegurado su lugar estratégico en la conformación de la industria. El mismo aporta la energía que requieren tanto la industria como los hogares. Asimismo, el servicio del sector eléctrico se presenta como uno de los mercados más sofisticados desde el punto de vista tecnológico, con la aparición de tecnologías que facilitan la productividad de las organizaciones. Su importancia relativa ha aumentado en las últimas tres décadas, pues se considera que su estructura organizativa y de mercado, y la expansión de su infraestructura cumplen un importante rol en la forma como la sociedad afronta retos complejos como los que se derivan del cambio climático. Si bien existe un conjunto de agentes claramente delimitados, reglas de juego aparentemente transparentes y un proceso de intercambio semicentralizado, el sector tiene una de las barreras de acceso más consolidadas, lo que favorece la conformación de monopolios de industria. Como tal, es innegable su desarrollo reciente.

Pese a lo anterior, el sector eléctrico viene presentando, sobre todo en la última década, síntomas preocupantes que ponen en tela de juicio las acciones que han llevado a cabo tanto el Estado como los privados y los actores institucionales. En efecto, eventos como las secuelas recurrentes de los fenómenos de El Niño y La Niña, el valor cada vez más alto de la prestación del servicio, la disminución de la autonomía y la autosuficiencia energéticas, la venta de Isagén y los escándalos en la prestación del servicio, ponen en tela de juicio sus avances. El sector, entonces, si bien presenta una modernización recurrente, al mismo tiempo parece favorecer una estructura en la que sus beneficios no se socializan claramente a toda la población, incluso poniendo en jaque la estabilidad operativa del sistema.

En ese marco se desarrolla el presente trabajo. Nuestro interés es llamar la atención sobre un conjunto de fenómenos que se presentan como desafíos y que deben preocupar no solo a los agentes del sistema, sino a todos los ciudadanos. Incluso, haciendo énfasis en que el lugar que otorga el sistema a la ciudadanía es de orden marginal, como sujetos pasivos ante la gestión organizacional y estratégica de los elementos del sector.

El documento hace una descripción de algunos eventos significativos que, observados desde una óptica amplia, permiten establecer el panorama que enfrentan estas instituciones. Para ello, se presenta una breve reseña del contexto de análisis y se explica la metodología. Posteriormente, se expone 


\section{Contexto Leon-alme, F}

cada uno de los puntos que se consideran críticos para pensar el desarrollo futuro del sector eléctrico. Como conclusión, se indica que el desarrollo del sector requiere una intervención importante que rescate el papel de lo político y que ayude a concretar los objetivos de expansión, calidad y eficiencia del sistema. Estas conclusiones se derivan de la primera fase de indagación de una investigación que analiza el proceso de implementación de las Normas Internacionales de Información Financiera en el sector eléctrico.

\section{Contexto de análisis}

En Colombia, los orígenes del sector eléctrico se ubican a finales del siglo XIX, época en la cual existían algunas empresas de carácter privado que se dedicaban a las cuatro actividades que componen la cadena productiva del sector (Figura 1). Durante la primera mitad del siglo XX, el sector tuvo un alto crecimiento derivado de los siguientes factores: i) crecimiento de las ciudades, ii) desarrollo de nuevas tecnologías y iii) tendencia creciente en la demanda por parte de los hogares (García, 2006).

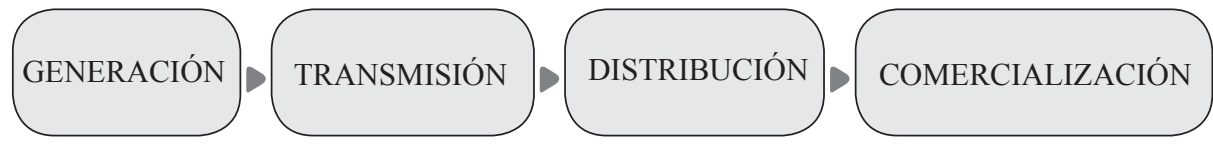

Figura 1. Cadena productiva de la energía eléctrica.

Elaboración propia con base en García (2006), Rodríguez (2010) y CREG (2016).

En la segunda mitad de la década de los ochenta, se identifica una brecha entre los recursos requeridos para inversión y la generación propia de fondos, ya que los ingresos provenientes de las tarifas no eran suficientes para cubrir los costos de inversión y funcionamiento; para enfrentar esta situación, se recurrió a la obtención de recursos del presupuesto público (García, 2006).

Lo anterior pudo ocurrir como resultado de algunos hechos de carácter internacional que se presentaron en las décadas de los setenta y los ochenta como son: i) recesión económica mundial, ii) aumento en los precios del petróleo y iii) crisis internacional de la deuda. A inicios de los noventa, en un diagnóstico de las empresas de energía estatales, se concluyó que presentaban ineficiencia operativa, administrativa y financiera (CREG, 2016).

Derivado de los elementos descritos y como resultado de la implementación de las políticas que caracterizan el Consenso de Washington, proceso realizado en Colombia en la primera mitad de la década de los noventa, se efectuaron algunas reformas en el sector que básicamente buscaban la privatización de 
las compañías de servicios públicos -las cuales hasta ese momento eran en su mayoría propiedad del Estado-y la promoción de la libre competencia.

En ese contexto se pueden identificar dos hechos que marcarían el rumbo del sistema: i) la Constitución Política de Colombia, que buscaba impulsar la eficiencia y la competitividad, y ii) las sequías provocadas por el fenómeno de El Niño en el año 1991 que llevaron al racionamiento de energía entre 1991 y 1992 (García, 2006; CREG, 2016).

En las últimas dos décadas se observa un proceso importante de consolidación de un mercado regulado en el que los diferentes agentes pueden tomar parte tanto en la producción, la distribución y la comercialización como en actividades independientes. El objeto de estas reformas fue consolidar la expansión del sistema, crear un sistema de precios eficientes y enfrentar los efectos del fenómeno de El Niño, lo cual permitió, en ese marco, la consolidación de una serie de entidades que fueron ganando peso estratégico y que con el tiempo se convirtieron en dominantes, donde el papel de los productores y transportadores de energía llegaría a ser definitivo. De esta forma se estructuró un sector que se encuentra determinado por un conjunto de instituciones que colaboran con su adecuado funcionamiento. En ese sentido, como se ilustra en la Figura 2, se identifican cuatro factores clave: i) política, ii) regulación, iii) mercado y iv) supervisión y control.

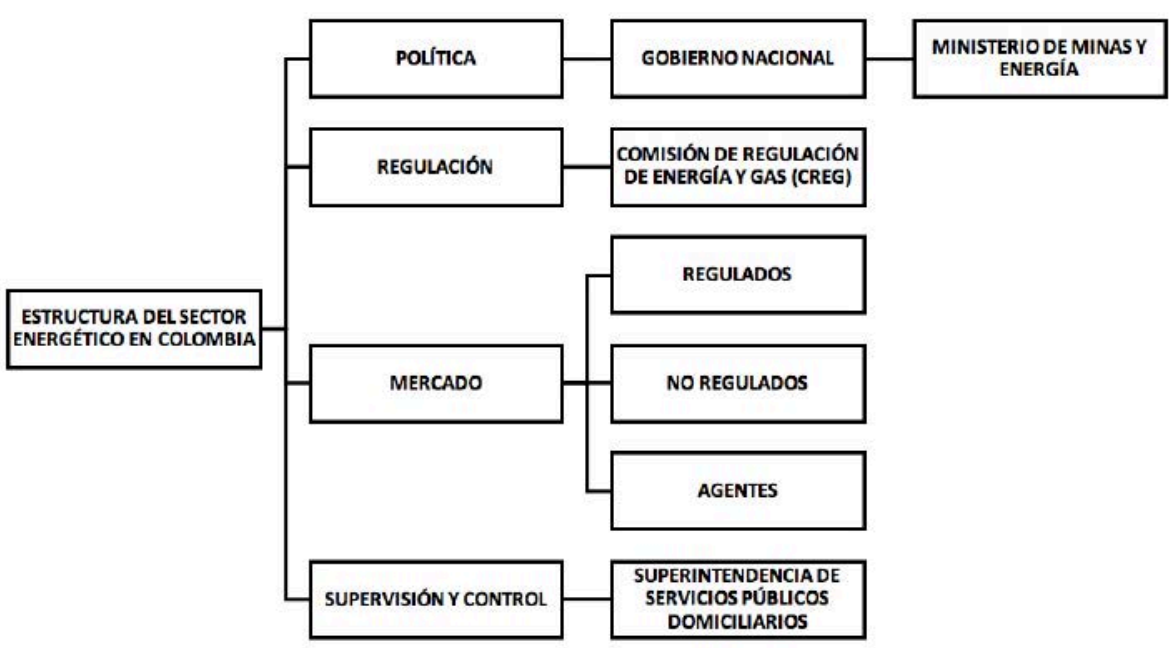

Figura 2. Estructura del sector eléctrico colombiano.

Elaboración propia con base en CREG (2016) y OLADE (2013). 


\section{Contexto Leon-aime, E}

Teniendo en cuenta lo anterior, se propuso que se reconocieran los costos asumidos por el sistema y que se lograra una estructura de precios que favoreciera la modernización y estabilidad del sistema. Esto incluyó la creación de cargos de capacidad y confiabilidad que tenían como objetivo, por un lado, hacer sostenible la expansión del sistema y, por el otro, crear un sistema de reserva que protegiera a los consumidores de los efectos del fenómeno de El Niño.

Como resultado de este esquema, a finales de la década de los noventa, se generó una transformación que privilegió la conformación de hidroeléctricas como eje del proceso de producción, y un parque de reserva asociado a la construcción de plantas térmicas. Se consideró, entonces, que con esta estrategia el sistema se protegería de los fenómenos climáticos y sería relativamente suficiente. El país incluso se proyectó como un exportador energético, llevando los procesos de interconexión a los países vecinos (Ecuador, Venezuela y Panamá). Los excedentes del sistema también permitieron que algunas empresas como Isagén y la Empresa de Energía de Bogotá se arriesgaran a realizar inversiones en proyectos de generación y transporte de energía en otros países suramericanos. La percepción global del funcionamiento del sistema fue relativamente positiva. En particular, el sector fue un referente de transformación institucional replicable, donde diversos actores salieron fortalecidos y el mercado regulado parecía cumplir sus propósitos. Algunas empresas como ISA, la EEB o EPM se convirtieron en referentes regionales, y tuvieron un peso importante no solo en los procesos de modernización del servicio, sino en la manera como asumían la regulación.

En los últimos cinco años (2012-2017) la participación del mercado regulado en la demanda real ha aumentado $(2,7 \%)$, mientras que la del mercado no regulado y la de los consumos del sector han disminuido ( $1,5 \%$ y $1,2 \%$, respectivamente). En lo corrido del 2017, el mercado regulado ha tenido una participación en la demanda real de $68,4 \%$, mientras que la del mercado no regulado ha sido de $31,1 \%$ y la de los consumos de $0,5 \%$ (Figura 3 ). 


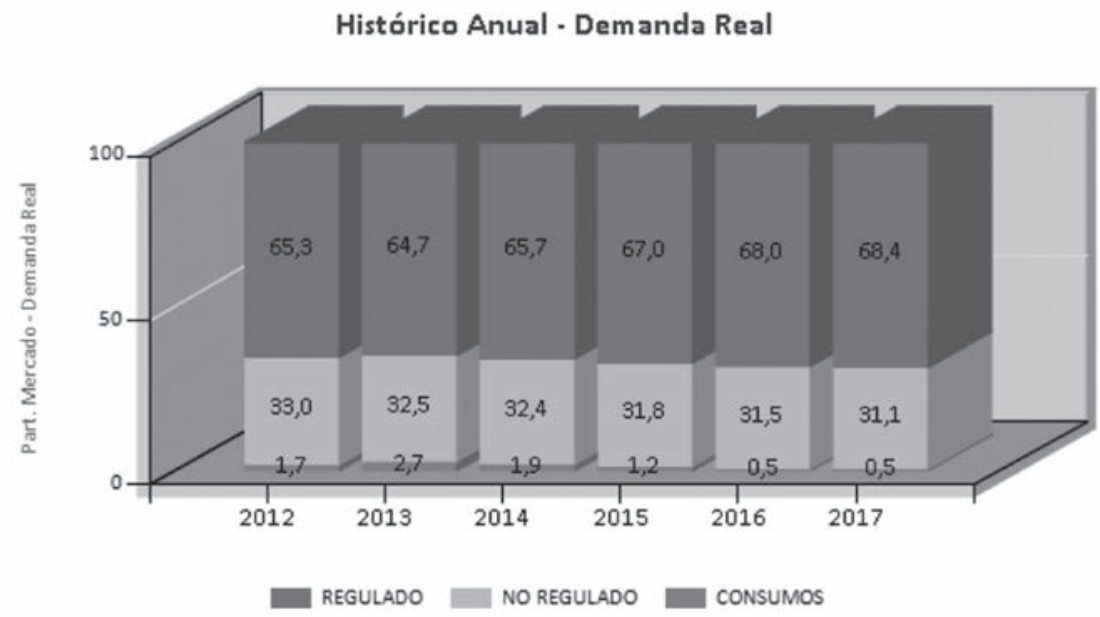

Figura 3. Participación en la demanda real (2012-2017).

SIEL (2017).

Por otro lado, el comportamiento del precio promedio en Bolsa en el periodo 2012-2015 fue de continuo aumento (UPME, 2016), mientras que en el transcurso de 2016 la evolución del precio promedio de los contratos de energía se mantuvo relativamente estable, y el precio promedio en la Bolsa Nacional de Energía presentó aumentos significativos en los primeros meses del año 2017 (SIEL, 2017). Recientemente se ha observado que la capacidad efectiva de generación ha tenido ajustes importantes, si bien su aumento se ha ralentizado en los últimos años.

Tabla 1.

Indicadores de generación, capacidad y evolución del precio de bolsa

Indicadores de generación y capacidad Indicadores de evolución del precio en bolsa

\begin{tabular}{lll}
\hline - & Capacidad Efectiva Neta (MV) & Precio Bolsa Nacional (\$/KWh) \\
Total acumulado 2017 (al 8-Junio): & Total acumulado 2017 (al 5-Junio): \\
16.689,038 & $\begin{array}{l}\text { 105,71 } \\
\text { Total acumulado año 2016: 16.597,018 }\end{array}$ & Total acumulado año 2016: 308,89 \\
& \\
Generación (GWh) & Precio Promedio Contrato (\$/KWh) \\
Total acumulado 2017 (al 8-Junio): & Total acumulado 2017 (al 5-Junio): \\
28.690,730 & 166,82 \\
Total acumulado año 2016: 65.935,243 & Total acumulado año 2016: 156,25 \\
\hline
\end{tabular}

Elaboración propia con base en SIEL (2017). 


\section{Contexto Loon-aime, F}

Pese a ello, la capacidad ha sido cuestionada, ya que si bien se mantiene cierta regularidad, el fenómeno de El Niño de finales de 2015 e inicios de 2016 significó una coyuntura importante que llevó a que el país llegara a situaciones significativas de importación de energía, pues, si bien la estructura de mercado estaba diseñada para utilizar la capacidad de reserva fundada en la utilización de energía térmica, esta resultó insuficiente para hacer frente a este fenómeno. Como resultado de lo anterior, desde el mes de noviembre de 2016 el país restringió sus exportaciones apoyado en la autogeneración y la importación de energía (ver Figura 4).

De la misma manera, la estrategia marcada en el sector eléctrico se ha concentrado en la producción de energía de origen hidráulico. Con ello, la capacidad de generación se ha incrementado en términos globales gracias a la expansión de este tipo de energía, no obstante, la capacidad de generación del sistema de reserva basado en energía térmica sustentada en gas ha tenido ajustes a la baja.
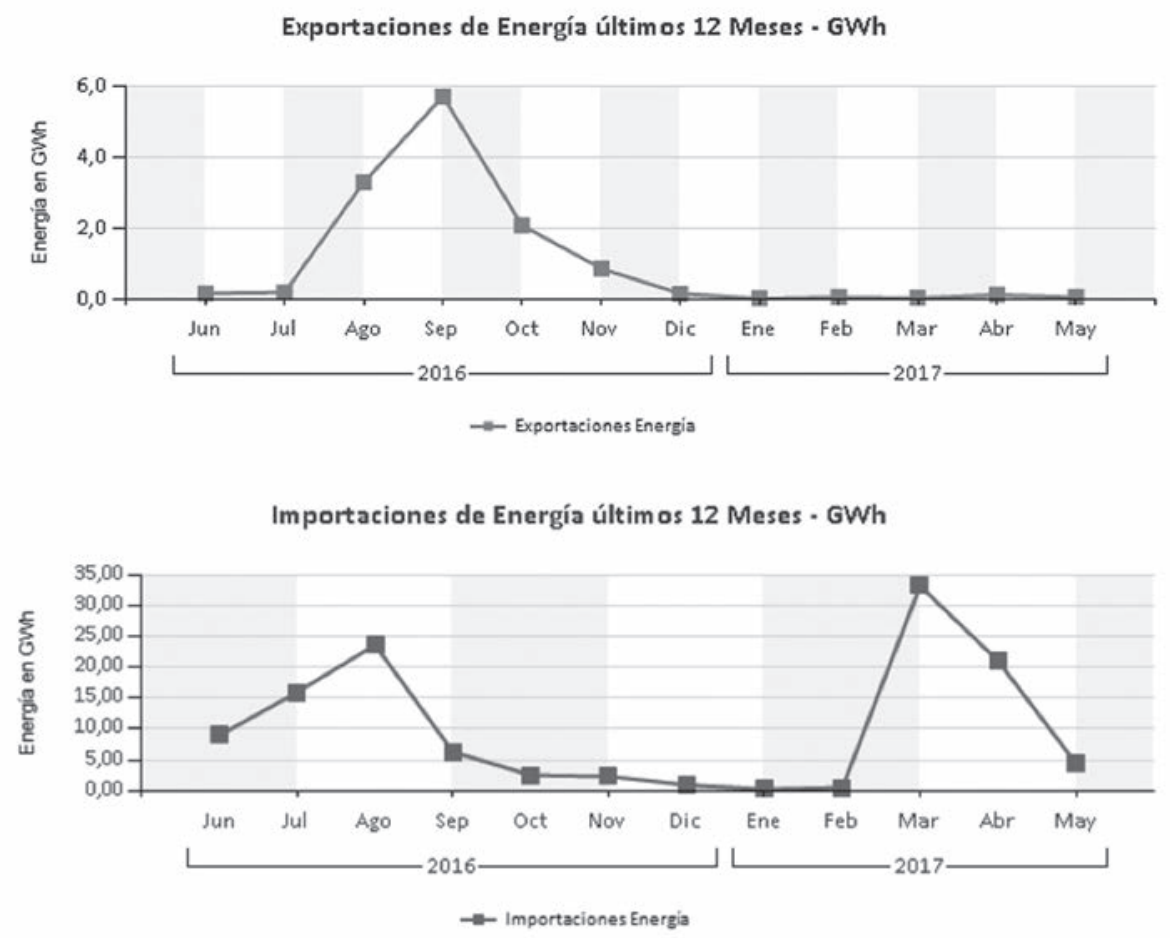

Figura 4. Exportaciones e importaciones de energía (2016-2017).

SIEL (2017). 
De acuerdo con el Sistema de Información Eléctrico Colombiano (SIEL, 2017), si bien el gas natural mantiene la segunda mayor participación en la generación eléctrica $(16,69 \%)$, en los últimos años se ha privilegiado el carbón frente al gas natural; en el periodo 2012-2016 su participación aumentó 4,04\% frente a un aumento del gas natural en 1,33\%. Lo anterior se explica, en parte, por el aumento de los precios de los derivados del petróleo en la última década, lo que contrasta con la baja promoción de energías alternativas.

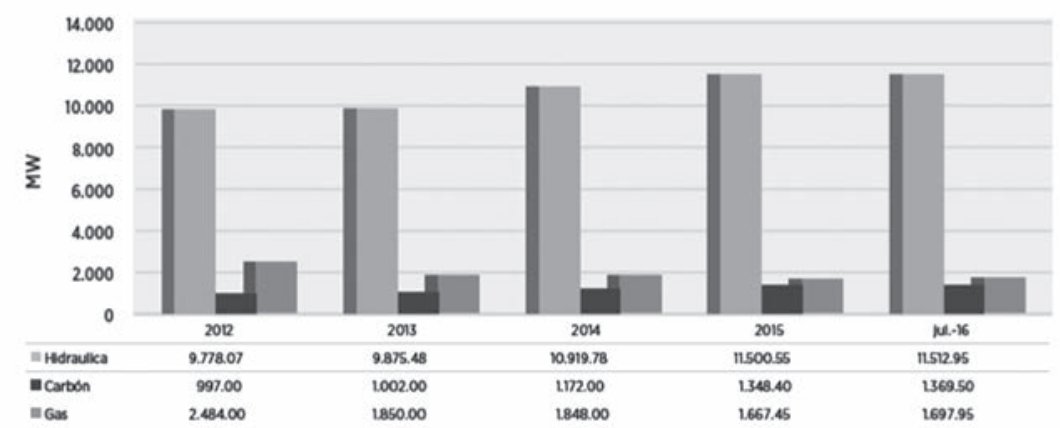

Figura 5. Capacidad efectiva de generación (2012-2016).

UPME (2016, p.17).

Recientemente el sector también ha recibido el impacto de la fallida intervención sobre Electricaribe, lo cual ha puesto de manifiesto la vulnerabilidad del sistema frente a los operadores y ha creado, al mismo tiempo, tanto problemas en la forma como la ciudadanía percibe la gestión de las empresas del sector, como incertidumbre en el objetivo que persigue la inversión privada en este tipo de organizaciones. El desarrollo de este caso ha tenido como eje la búsqueda de salidas que permitan, o bien hallar un inversor estratégico, o bien consolidar el proceso de estatización de la empresa. A esto se suma la reciente privatización de Isagén que provocó malestar en algunos sectores, y que junto al caso de Reficar significó un proceso de cuestionamiento permanente al Gobierno por la cesación estratégica de ese activo, por la utilización de los recursos derivados de la venta de un activo para financiar otro, y por el efecto que puede tener a futuro este negocio en la expansión del sistema interconectado. 


\section{Contexto Leon-aime, F}

\section{Metodología}

La investigación se realizó mediante la revisión documental de una base de datos construida para examinar el comportamiento organizacional de las empresas del sector eléctrico sometidas a la Resolución 743 de 2013 de la Contaduría General de la Nación, donde se contempla la adopción de las Normas Internacionales de Información Financiera (NIIF). En ese marco se consideró importante hacer un proceso de análisis comprensivo del contexto al que se enfrentan estas empresas, escenario en el que surge este documento.

Teniendo como punto de partida un enfoque descriptivo comprensivo, la investigación realiza un proceso de sistematización de información para llegar a la construcción de la síntesis aquí presente. Se recuperaron datos de tipo cuantitativo y cualitativo de la información institucional de las empresas objeto de análisis:

- Isagén S. A.

- Intercolombia S. A

- Centrales Eléctricas del Norte de Santander S. A.

- Central Hidroeléctrica de Caldas S. A.

- Empresa de Energía de Cundinamarca S. A

- Distribuidora Eléctrica de Cundinamarca S. A.

- Empresa de Energía de Bogotá S. A.

- Electrificadora de Santander S. A.

- Empresa de Energía del Quindío S. A.

- Interconexión Eléctrica S. A.

- Transelca S. A.

También se incluyó información de la Superintendencia de Servicios Públicos Domiciliarios, el Ministerio de Minas y Energía, la Comisión de Regulación de Energía y Gas (CREG), la Unidad de Planeación Minero Energética (UPME), el Sistema de Información Eléctrico Colombiano (SIEL), para los últimos 5 años. La base documental integró la revisión y sistematización de informes de gestión, planes de expansión, informes consolidados, información contable, notas a los informes financieros, etc. Para este artículo se ha utilizado parcialmente esta base, prestando atención a aquellos documentos que han aportado a la síntesis que se presenta a continuación. 


\section{Resultados}

\section{Discusión: un panorama de desafíos}

La revisión elaborada permitió identificar seis elementos que pueden ser determinantes en el desarrollo del sector eléctrico colombiano a futuro. Tales temáticas generan señales que deben ser tenidas en cuenta, en tanto son el resultado de una estructura que ha privilegiado formas de gestión de un activo público. Los retos posteriores son indicadores de situaciones complejas en las que el papel de la ciudadanía será demandado.

\section{La concentración de la propiedad de los activos del sistema}

Desde la estructuración del sistema institucional de funcionamiento en los años noventa se agenció un proceso que buscó aumentar la capacidad organizacional de los prestadores del servicio, en sus tres dimensiones: generación, transmisión y distribución. A partir de ese momento se planteó que para mejorar la eficiencia del sistema, era esencial una estructura de mercado basada en la existencia de organizaciones empresariales publico/privadas como sociedades por acciones. Ese fue el espíritu de la Ley 142 de 1994, donde se definen el servicio y las estructuras societarias de las empresas. De la misma manera, con la Ley 143 de 1994 se dio forma al régimen de generación, interconexión, transmisión, distribución y comercialización de electricidad, lo cual obligó a municipalidades, departamentos y a la Nación a reordenar estratégicamente estas empresas. Muchas de ellas se convirtieron en sociedades públicas por acciones, otras se transformaron en sociedades mixtas, y otras fueron privatizadas. La entrada del capital privado, en ese momento de fuente externa, se consideró como un proceso estratégico para financiar la expansión del sistema.

A partir de allí se configuraron una serie de relaciones mediante las cuales algunas organizaciones empezaron a evidenciar un peso relativo más importante frente a otras en los mercados recientemente establecidos. Paulatinamente, las empresas generadoras que poseían activos estratégicos de gran dimensión en la producción de electricidad se fueron haciendo dominantes, no solo por el punto de partida inicial, sino porque al participar tanto del sistema interconectado como del proceso de contratación paralelo lograron reunir los recursos suficientes para poder establecer nuevos proyectos de expansión. La expansión entonces fue sinónimo de poder, y tal poder con el tiempo favoreció la concentración. En la primera década del siglo XXI estos procesos de expansión tuvieron el apoyo de la inversión pública y privada, lo cual llevó a que un conjunto de pequeñas generadoras quedaran por fuera del circuito de los recursos disponibles. El 


\section{Contexto Leon-aime, E}

número de empresas se redujo de manera significativa, ya que las dominantes empezaron a adquirir o fusionarse con operadores públicos de menor tamaño.

En el plano de la interconexión, el mercado se montó bajo la estructura de un solo operador que interconectaba productores y comercializadores de energía, de este modo, la estrategia del Gobierno nacional se centró en la expansión del papel de ISA e Isagén, cuyo rol fue muy importante, ya que al concebirse como actores públicos del orden nacional, se consideró que tenían la capacidad de ocupar un lugar relevante en la regulación y las relaciones de los agentes. Las compras de energía eran capitalizadas en la transmisión, con lo cual su papel se incluía en los contratos y fortalecían a las entidades. La expansión del sistema era solo posible en función de estos actores, de manera que los nuevos proyectos debían contar con su participación, para que así estuvieran vinculados al sistema. Así, los activos de transmisión se monopolizaron.

En el caso de la distribución el panorama fue mucho más complejo. En términos generales, se tendió a la conformación de sociedades mixtas en las que el número de suscriptores fue un botín significativo. Las tensiones de la política regional tendrían un impacto importante en la gestión de estas organizaciones. Mientras en algunos contextos institucionales abogaron por la conformación de estructuras autónomas tecnocráticas (por ejemplo, Medellín con EPM y Bogotá con Codensa) que fortalecieron las empresas y mantuvieron una estrategia clara por la calidad del servicio, en otros contextos el poder regional se impuso y se extendió a estas entidades, haciendo que presentaran dificultades para la sostenibilidad financiera y la prestación del servicio (por ejemplo Emcali, en el Valle del Cauca, y Electricaribe).

Tabla 2.

Algunos eventos de la concentración de activos

\begin{tabular}{lc}
\hline \multicolumn{1}{c}{ Evento } & Fuente \\
\hline $\begin{array}{l}\text { Para 2009, Isa era la mayor empresa de transporte de energía } \\
\text { eléctrica en Colombia y la única con cubrimiento nacional, con una }\end{array}$ & \\
infraestructura de 10.000 km de circuitos de transmisión con tensión & Ministerio de Minas y \\
superior a $115 \mathrm{kV}, 57$ subestaciones, 12.672 megavoltaamperios & Energía (2009). \\
(MVA) de transformación y 4.177 megavoltaamperios reactivos & \\
(MVAR) de compensación reactiva. & \\
\hline A diciembre de 2012, el sector eléctrico colombiano estaba compuesto & \\
por 92 agentes comercializadores (80 en 2011), 50 agentes generadores & UPME (2013) \\
(53 en 2011) y 30 distribuidores (32 en 2011). & \\
\hline
\end{tabular}




\begin{tabular}{lc}
\hline Evento & Fuente \\
\hline A diciembre de 2012, cinco agentes contaban con 76\% de la capacidad & \\
efectiva neta de generación, así: Emgesa (2.880 MW), EPM (2.600 & UPME (2013) \\
MW), Isagen (2.100 MW), Gecelca (1.380 MW) y Epsa (1.070 MW). & \\
\hline
\end{tabular}

Los cambios constitucionales permitieron el ingreso al sector de nuevos oferentes del servicio de energía, motivo por el cual se presentaron grandes procesos de privatización, lo que llevó al Estado

CREG (2016) a concentrarse en las actividades de regulación, control y vigilancia.

Se presentó una variación nula en la cantidad de agentes comercializadores durante los años 2013 y 2014.

UPME (2015)

La Ley 143 de 1994 segmentó el servicio de energía eléctrica en cuatro actividades: generación, transmisión, distribución y comercialización, las cuales pueden ser desarrolladas por empresas independientes.

CHEC Grupo EPM (2016)

El $70 \%$ de la generación real de 2010 fue suministrada por cuatro agentes generadores: Empresas Públicas de Medellín ESP (EPM), Emgesa S. A. ESP, Isagén S. A. ESP, y la Generadora y Comercializadora de energía del Caribe S. A. ESP (Gecelca).

Superintendencia de Industria y Comercio (2011).

Elaboración propia.

\section{El lento crecimiento de la capacidad instalada}

Como previamente se había planteado, se vive un proceso de ralentización de la capacidad instalada. Si bien a principios de la década anterior se prestó atención a la generación de nuevos proyectos, este proceso entró en declive finalizando la década. Buena parte de esto se explica por el despliegue de una estrategia que buscó mantener el lugar de predominio de los generadores de energía tradicionales.

La energía basada en el poder hidráulico se convirtió en el referente para hacer frente a los problemas de expansión en los años noventa. En ese momento se la consideró la mejor alternativa, ya que se concebía que los recursos hídricos eran de fácil obtención y se tenía una tradición de proyectos heredada del desarrollismo impulsado por los entes multilaterales. El Gobierno nacional y algunos gobiernos locales habían realizado inversiones importantes en este sentido. De tal modo, la expansión se configuró como un proceso de prevalencia de la potencia sobre otros.

Teniendo esto como eje, el sistema de mercado establecido y sus reglas de juego les permitieron a las generadoras asegurarse un lugar esencial en el despliegue de nuevos proyectos. Las expansiones tenían que ver con un aumento de la capacidad de generación en lugares existentes o con la construcción de nuevos 


\section{Contexto Leon-paime, F}

proyectos en contextos nuevos. El potencial hídrico se concibió para apropiarse al máximo potencial, ya que ofrecía cierto nivel de independencia de los combustibles fósiles y se consideró que aseguraba la estabilidad de los precios.

Los proyectos de este tipo empezaron a tener inconvenientes derivados, en primer lugar, del efecto de los fenómenos climáticos y, en segundo lugar, del tamaño de las inversiones que tenían que asumir. En ese sentido, primero se creó un esquema de remuneración que asegurara dar cuenta de estos fenómenos, que derivó en el cargo de capacidad (1994) que posteriormente se convertiría en el cargo de confiabilidad (2006). Se buscó que los proveedores contaran con recursos que les otorgaran herramientas para proveer energía en momentos críticos de la capacidad hídrica. Este esquema funcionó bien, porque aseguraba remuneraciones para los productores incluso en momentos críticos, ya que al asociarlo al "precio de escasez" proporcionaba los incentivos adecuados para que la expansión se mantuviera.

Pese a lo anterior, los fenómenos de El Niño recientes pusieron en evidencia que las inversiones para la expansión necesaria eran insuficientes. Las generadoras no lograron enfrentar las crisis y el país ha pasado de productor a importador reiterado de energía. Los cargos creados como remuneración han sido cuestionados, puesto que si bien este costo fue trasladado al consumidor primario, y transferido de los comercializadores a los generadores, la expansión parece no alcanzar a suplir la demanda necesaria. Asimismo, hay dudas sobre la cantidad de recursos recaudados y las inversiones realizadas por parte de los generadores, e incluso se cuestiona si las mismas generadoras pueden estar utilizando una estrategia inversa para crear comportamientos de producción que las acerquen a los precios de escasez. Esta situación se encuentra en debate permanente, sin que se hayan introducido cambios significativos en los últimos años.

Finalmente, algunas instituciones del sector, en especial las generadoras, se quejan de que encuentran barreras institucionales para llevar adelante la expansión. En particular, su queja permanente tiene que ver con el poder de las comunidades, los requisitos para las nuevas infraestructuras y los protocolos para obtener licencias ambientales. Se genera un choque porque los nuevos proyectos de expansión tienen que hacer frente a la presión pública. En ese contexto, los últimos gobiernos se han mostrado más favorables a flexibilizar los procesos, con el fin de cumplir con los plazos de intervención y de financiación de la infraestructura, lo cual ha significado el descontento de muchas comunidades. Las instituciones han justificado la ralentización del proceso de expansión, en parte, por el proceso jurídico, técnico y consultivo que tienen los nuevos proyectos. 
Tabla 3.

Eventos relacionados con la expansión del sistema de generación

\begin{tabular}{|c|c|}
\hline Evento & Fuente \\
\hline $\begin{array}{l}\text { Se identifican dificultades para ampliar y renovar la red de transmisión } \\
\text { por factores tales como costos, oposición a su construcción, impactos } \\
\text { generados, etc. En ese sentido, se busca la generación de energía de manera } \\
\text { descentralizada y cerca de los puntos de consumo, lo cual aumentará la } \\
\text { cantidad de participantes en el mercado, demandando con ello nuevas } \\
\text { formas de operación y de conexión de los sistemas eléctricos. }\end{array}$ & UPME (2015) \\
\hline $\begin{array}{l}\text { La capacidad instalada se puede afectar por: i) mantenimiento, ii) } \\
\text { pruebas, iii) uso de combustible alterno, iv) nivel de los embalses. }\end{array}$ & $\begin{array}{l}\text { Ministerio de Minas } \\
\text { y Energía (2009) }\end{array}$ \\
\hline $\begin{array}{l}\text { El mercado colombiano se caracteriza por: i) vulnerabilidad } \\
\text { hidrológica, ii) baja capacidad de regulación de los embalses, iii) } \\
\text { aversión al racionamiento. }\end{array}$ & $\begin{array}{l}\text { Barrera y García } \\
\quad(2010)\end{array}$ \\
\hline $\begin{array}{l}\text { El crecimiento de la capacidad instalada entre } 1993 \text { y } 2013 \text { fue de } \\
46,58 \% \text { hasta llegar a } 14.426,5 \mathrm{MW} \text {. }\end{array}$ & UPME (2013) \\
\hline $\begin{array}{l}\text { Para 2014, la capacidad instalada se mantuvo muy similar a la del año } \\
\text { anterior en términos de magnitud, pero se modificó la participación por } \\
\text { tipo de tecnología. }\end{array}$ & UPME (2015) \\
\hline
\end{tabular}

Durante el 2014 entraron en operación algunos proyectos hidroeléctricos tales como Sogamoso (800 MW) y El Quimbo (420 MW), los cuales aportaron generación con mayor economía en el uso de recursos, lo que permitió reducir el costo marginal durante los meses de febrero a UPME (2015) diciembre.

La capacidad instalada del sistema eléctrico nacional se ha incrementado gradualmente en los últimos 20 años, gracias a iniciativas públicas y privadas para el desarrollo de nuevas plantas de generación.

UPME (2013)

Frente a la meta propuesta en el plan de desarrollo de incrementar la prestación del servicio de energía eléctrica a 24 horas en 33 cabeceras municipales, a diciembre de 2012 se había cumplido con este propósito UPME (2014) en 8 cabeceras.

Teniendo en cuenta estos resultados, el registro de proyectos de la UPME y las plantas que actualmente están en construcción, se plantea para el año 2022 la entrada en operación de un proyecto térmico de 300 MW a base de carbón, dos proyectos hidroeléctricos para el año 2025 de 400 y 700 MW cada uno, y dos cierres de ciclo en la Costa Atlántica de $250 \mathrm{MW}$, los cuales deben estar en servicio en el 2022 y 2025 (se considera también la segunda etapa de Ituango con 1.200 MW UPME (2013) de capacidad). Este escenario plasma las condiciones que debieran suceder en el país en cuanto a generación. Así mismo, conserva la tendencia presentada en el Cargo por Confiabilidad, en el sentido que el sistema se expande con aquellos recursos de los cuales se tiene mayor disponibilidad.

\section{Elaboración propia.}




\section{Contexto Loonn-aime, F}

\section{La difícil incorporación de las tecnologías limpias}

Las condiciones que se generaron como consecuencia de la estrategia de prevalencia de energías basadas en el potencial hídrico con un sistema de reserva orientado al ámbito térmico, han atrasado la entrada de generación de energías limpias al país. La estructura de remuneración de las productoras tradicionales hizo que estas no tuvieran los incentivos suficientes para la temprana explotación de estos tipos de energías. Por el contrario, durante las primeras dos décadas, se entendió que estas energías (eólica o solar) no tenían posibilidades de hacer parte de los procesos de expansión, y que podrían incorporarse en el sistema de generación de reserva a través de la generación térmica a partir de biomasa. Este orden presente en la regulación dejó por fuera la posibilidad de que aparecieran nuevos generadores durante un tiempo significativo. Los diferentes proyectos se encontraron con el poco interés de los generadores tradicionales, y también con el poco espacio para que emergieran oferentes nuevos. El sistema privilegió entonces, y durante largo tiempo, a los monopolios de la generación ya establecidos.

Algunos procesos de autoproducción fueron apareciendo en el horizonte. Estos se hicieron viables con el aumento relativo de los precios de la energía, lo cual puso a actores de la industria nacional a pensar en realizar inversiones propias para el autoconsumo. Si bien se tenía la experiencia de contratos de producción exclusiva, se entendió que estos no eran suficientemente costoeficientes para las demandas de expansión del sector privado. La inversión en energías alternativas se va abriendo paso en el ámbito empresarial, pues si bien se observa como una inversión importante, se empieza a percibir que es sostenible a largo plazo, dado el aumento del costo de la energía en el mercado nacional y también el aumento de los precios en el mercado nacional e internacional de los combustibles fósiles. El empresariado ve entonces en este tipo de inversión una estrategia económica que también le da autonomía frente al sistema interconectado.

Pese a lo anterior, y aunque durante los últimos años se han sacado adelante nuevas regulaciones que abogan por los incentivos tributarios para este tipo de inversiones, todavía se encuentra en desarrollo la posibilidad de que se genere un mercado amplio de pequeños generadores asociados al sistema interconectado. Al mismo tiempo, el autoconsumo residencial está en fases muy preliminares, con costos de inversión muy altos, y con la ausencia de incentivos por parte del sistema general. 
Como consecuencia de lo anterior, el país está atrasado con relación a otros países en el desarrollo de proyectos de gran escala, por lo que la generación de energía proveniente de este tipo de recurso está en promedio entre el $2 \%$ y el 3\% del total nacional, y como previamente se indicó, en buena medida orientada al autoconsumo empresarial.

Tabla 4.

Eventos de la difícil incorporación de tecnologías limpias

\begin{tabular}{lc}
\hline \multicolumn{1}{c}{ Evento } & Fuente \\
\hline El aprovechamiento de energías renovables en el país para la & UPME (2013) \\
producción de electricidad inicia con la hidroenergía; actualmente & \\
este es el mayor recurso renovable del país y genera alrededor del & \\
$70 \%$ de la electricidad. & \\
& \\
\hline Al analizar la evolución histórica por tecnología en la capacidad & UPME (2015) \\
instalada del Sistema Interconectado Nacional (SIN), se evidencia & \\
la prevalencia de plantas hidroeléctricas, las cuales aumentaron & \\
notoriamente su capacidad con la entrada del proyecto Sogamoso & \\
en el segundo semestre de 2014. Por otro lado, la participación & \\
de plantas térmicas, tanto de gas como de carbón y combustibles & \\
líquidos, ha tenido modificaciones debido a que la capacidad se & \\
calcula con el combustible principal y los contratos de respaldo & \\
para su suministro. &
\end{tabular}

La posibilidad de generar energía mediante fuentes alternativas tales como plantas eólicas, generación solar fotovoltaica, geotermia UPME (2015) y biomasa, toma mayor valor cuando se presentan periodos de sequía, tales como el causado por el fenómeno de El Niño, ya que en esos casos se reduce la capacidad productiva de los generadores hidroeléctricos.

\begin{tabular}{l}
\hline $\begin{array}{l}\text { Para el desarrollo de Redes Inteligentes (RI) se requiere una } \\
\text { transformación del mercado eléctrico, lo cual demanda cambios } \\
\text { institucionales así como de regulación. }\end{array}$ \\
$\begin{array}{l}\text { La Matriz Energética colombiana tiene una capacidad } \\
\text { predominantemente hidráulica con un respaldo térmico que }\end{array}$ \\
$\begin{array}{l}\text { Asociación Colombiana } \\
\text { de Generadores de } \\
\text { confiabilidad del } 99,93 \% \text {. }\end{array}$
\end{tabular}

El registro de proyectos de la UPME comienza a reflejar un mayor UPME (2013) interés por implementar nuevas e innovadoras tecnologías de generación de electricidad. Los agentes han manifestado el deseo de invertir en fuentes no convencionales de energía (FNCE) para la generación de electricidad.

Elaboración propia. 


\section{Contexto Leon-aime, F}

\section{La estructura del mercado impide la aparición de nuevos actores}

La aparición de nuevos actores es un fenómeno difícil tanto para el sector regulado como para el proceso de autoconsumo. El tamaño de las inversiones de expansión requiere un apoyo financiero importante. Esta situación hace que las instituciones que quieran entrar al mercado regulado encuentren inconvenientes, ya que el grado de riesgo de inversión es alto, y a no ser que se asocien con los productores o distribuidores ya existentes, no hay una posibilidad clara de nuevas inversiones. Asimismo, sectores como el solidario, el cooperativo y/o comunitario no han encontrado estructuras de regulación suficientemente sólidas para realizar inversiones.

En los últimos años, la aparición de proyectos de expansión asociados a energías renovables se ha concentrado en proyectos de impacto marginal en el sistema. En ellos el componente técnico se involucra a pequeñas empresas que, como tal, presentan soluciones para ámbitos específicos pero que no están orientadas a la competencia o a hacer parte del mercado interconectado. Mientras la interconexión no permita que pequeños agentes puedan vender energía, los estímulos para su desarrollo son bajos, ya que el fundamento de estos pequeños productores es poner al servicio del sistema excedentes energéticos que puedan ayudar a compensar los efectos del cambio climático.

La estructura de mercado está dominada por los agentes consolidados, y estos tienen incentivos suficientes para no realizar cambios significativos. Se observa entonces que grandes productores y distribuidores aseguran su posición dominante haciendo que el capital empresarial se oriente a proyectos de mediana envergadura.

Tabla 5 .

Dificultades para la aparición de nuevos actores

Evento

El funcionamiento del mercado eléctrico fue reglamentado con el fin de optimizar el recurso hídrico, sin embargo, las empresas privadas pertenecientes al sector no siguieron al pie de la letra estas reglas.
Fuente

Barrera y García

(2010)
El precio spot del mercado es factor fundamental para poder planear y proyectar la expansión del mercado eléctrico colombiano.
Barrera y García

(2010) 


\begin{tabular}{lc}
\hline Evento & Fuente \\
\hline $\begin{array}{l}\text { La desconfianza del sistema de precios es un problema para la operación } \\
\text { del cargo por confiabilidad y para el mercado colombiano. El cargo por } \\
\text { confiabilidad - y de hecho el mercado colombiano - está basado en la } \\
\text { premisa según la cual los precios dan las señales de producción y gestión } \\
\text { eficientes de los recursos escasos. En la medida en que no se tenga } \\
\text { confianza en la formación de precios pocas posibilidades existen de que } \\
\text { el mercado funcione o que las autoridades tengan la tranquilidad de dejar }\end{array}$ & $\begin{array}{c}\text { (2010) } \\
\text { la gestión del recurso a este mecanismo. }\end{array}$ \\
\hline $\begin{array}{l}\text { Durante el período comprendido entre los años 2010-2013, la regulación } \\
\text { normativa se enfocó en aspectos como la subasta de energía, medidas } \\
\text { para evitar el desabastecimiento y desarrollo de proyectos de gas natural. }\end{array}$ & $\begin{array}{c}\text { Ávila y Hurtado } \\
\text { (2016) }\end{array}$ \\
\hline
\end{tabular}

Las subastas realizadas durante los meses de mayo y junio de 2008 inicialmente fueron buenas para la expansión de proyectos, sin embargo, posteriormente ocurrieron sucesos que demostraron falencias en el Barrera y García mecanismo.

En la actualidad, todas las empresas que distribuyen energía también son comercializadoras. Sin embargo, no todas las empresas comercializadoras son distribuidoras. Los distribuidores de energía deben contribuir en el libre acceso de los usuarios a los SRT y SDL garantizando las condiciones de calidad y confiabilidad.

Superintendencia de Industria y Comercio (2011)

Elaboración propia.

\section{La ausencia de una estrategia clara frente al cambio climático}

El cambio climático es un desafío importante para todos los sectores estratégicos de la nación, no obstante, la ausencia de una política de Estado en este sentido es visible en el sector eléctrico. Si bien la estrategia se ha centrado en el recurso hídrico, el cual se considera un proceso de producción de alto impacto ambiental en la fase de construcción, pero de bajo impacto en el funcionamiento por la baja emisión de $\mathrm{CO}_{2}$, esto no responde a una estrategia frente al cambio climático sino a una tradición de producción que, como también lo afirmamos, se heredó del desarrollismo.

En ese sentido, ni el Gobierno nacional ni los gobiernos locales se han animado de manera decidida al desarrollo de proyectos de expansión basados en energías alternativas. Los trámites frente a entidades multilaterales para la financiación de este tipo de proyectos no han sido una preocupación central, a diferencia de otros países de la región (México, Perú, Chile) que han llevado a cabo proyectos de expansión con energías alternativas. 
La ausencia de una estrategia se da tanto en el lado de los productores como de los distribuidores. El despliegue de los cargos para la expansión no estuvo orientado para el uso de energías alternativas, de modo que la estabilidad del sistema no se aborda con este componente. Por lo tanto, los generadores tradicionales no ven la obligación ni el estímulo para el desarrollo de este tipo de proyectos. Al mismo tiempo, los distribuidores han tenido muy pocos avances al respecto, de tal manera que los esfuerzos han estado amarrados a promover la disminución del consumo, y a prácticas de ahorro y de sustitución de bombillería. Es así que existe un vacío importante en el desarrollo de una estrategia que ubique el sector energético frente al cambio climático, otorgando roles y responsabilidades tanto a generadores como a distribuidores.

Tabla 6 .

Eventos de ausencia de estrategias frente al cambio climático

Evento

Es necesario que se reconozca la tendencia verde, la cual se encuentra ligada a la generación mediante el uso de energías renovables y al uso más eficiente de la energía generada.

Las sequías provocadas por el fenómeno de El Niño en el año 1991 llevaron al racionamiento de energía entre 1991 y 1992.

La introducción del cargo por confiabilidad en el año 2006 ha sido un avance en la introducción de señales de mercado para el pago por "adequacy", pero su diseño tiene problemas. Al cargo se le criticó que siendo un único instrumento tuviese tantos objetivos, a saber: el objetivo de incentivar la entrada, el objetivo de servir como instrumento de confiabilidad todo el tiempo y, en especial, en épocas de hidrología crítica, y, finalmente, el objetivo de controlar el poder de mercado.

Si bien Colombia es un país rico en agua, también su alto relieve montañoso dificulta el aprovechamiento de la hidrografía.

A través de las políticas de regulación del sector térmico, se debe garantizar que se le atribuya la suficiente importancia en el marco de costos de razonamiento, dado que en caso que se presentara escasez generaría un impacto directo al servicio público domiciliario y sus precios.

La evolución de la demanda de energía se vio afectada para el periodo de enero 2011 a diciembre de 2012, debido al fenómeno de La Niña. Por las altas lluvias que se presentaban y las inundaciones era necesario que se generara desconexión de cargas.

Para el año 2021, el margen de reserva en un escenario de demanda alta estaría alrededor del $18 \%$, presentando una significativa reducción en comparación con años anteriores.
Fuente

SENA (2013)

García (2006); CREG

(2016)

Barrera y García (2010)

Barrera y García (2010)

Asociación

Colombiana de

Generadores de

Energía Eléctrica (2016)

UPME (2013)

(UPME, 2013)

Elaboración propia. 


\section{Estructuras de rendición de cuentas centradas en el inversor del sector institucional}

Uno de los aspectos menos trabajados, pero que tiene un papel importante en el desarrollo del sector eléctrico, son los procesos de rendición de cuentas, diseñados para satisfacer las necesidades informativas de los agentes institucionales del sector, pero no han sido orientados a satisfacer las necesidades de la ciudadanía. La conjunción institucional de superintendencias, comisiones de regulación, intermediarios, etc., se ha concentrado en generar sistemas de información útiles para el proceso de compra y venta de energía y dar cuenta de la capacidad y del estudio técnico de aspectos esenciales (por ejemplo, los informes de la Unidad de Planeación Minero Energética-UPME-).

Las estructuras de información y rendición de cuentas se concentran en el inversor del sector institucional. Por ejemplo, el sistema de información XM provee información a los integrantes del sistema, y el mismo es filial de ISA, que es transmisora de energía. Sin duda, esta es una poderosa herramienta para el monitoreo del mercado. Pero tales sistemas de información se enfocan en un tipo de agentes limitado, y dejan por fuera otro tipo de usuarios (académicos, comunitarios y ciudadanos). El reporte de información se concentra en el cumplimiento técnico y normativo de las entidades.

De la misma manera, la información clave que permita hacer seguimiento de proyectos, del uso de los cargos de confiabilidad, y de la forma de intervención en comunidades, es un aspecto que se deja por fuera de la información tradicional (aun aquella que incluye o se orienta a aspectos de responsabilidad social). En algunos casos, la información está dispersa y hace falta ser un experto para poder construir un panorama consolidado del proceso del sector.

Finalmente, no existe una estrategia de promoción de la participación ciudadana en el desarrollo del sector. Cuando se consulta a las comunidades, en algunos casos, estas son asumidas como un obstáculo para el desarrollo de nuevos proyectos. Se carece de estudios claros que permitan construir un programa de desarrollo del tipo de necesidades energéticas que tienen las comunidades. Asimismo, la relación de algunos distribuidores con sus consumidores es compleja, y más allá de las sanciones por prestación deficiente del servicio, no se involucran de manera activa con las comunidades.

En ese sentido, se configuró un espacio social donde los agentes del sector eléctrico se proyectan como actores autónomos que tienen derechos y obligaciones para sí mismos, pero que tienen poco interés en interactuar con las comunidades que, en algunos casos, han llegado a menospreciar (caso de 


\section{Contexto Leon-alme, F}

la extrapolación de la cultura de no pago, como responsable de la deficiente gestión financiera de algunos comercializadores).

Tabla 7.

Rendición de cuentas centradas en el inversor institucional

\begin{tabular}{lc}
\hline \multicolumn{1}{c}{ Evento } & Fuente \\
\hline $\begin{array}{l}\text { La Presidencia de la República, como más alta autoridad administrativa } \\
\text { en el sector eléctrico, delega la función en Minminas, quien además }\end{array}$ & $\begin{array}{c}\text { Superintendencia de } \\
\text { se apoya en CREG, UPME, Instituto de Promociones Energéticas y el }\end{array}$ \\
$\begin{array}{l}\text { Instituto de Investigación. } \\
\text { (2011) }\end{array}$ \\
\hline $\begin{array}{l}\text { La regulación se convierte entonces en un juego antagonista de suma } \\
\text { cero entre regulador y regulados y esa tensión se resuelve por medio } \\
\text { de sobrecostos para el sistema y los usuarios. }\end{array}$ & $\begin{array}{c}\text { Barrera y García } \\
\text { (2010) }\end{array}$ \\
\hline
\end{tabular}

Elaboración propia.

\section{Conclusiones}

Este documento ha explorado algunos de los principales desafíos a los que se enfrenta el sector eléctrico colombiano en los próximos años, el cual, como el documento lo evidencia, es el resultado de una serie de apuestas técnicas, políticas y de mercado que han configurado una estructura, que si bien ha satisfecho algunas de las problemáticas que se planteaban al principio de la década de los noventa del siglo anterior, éstas persisten o han derivado en condiciones que restan competitividad al mismo sector.

En ese sentido, el sector que se caracterizó por su profundo proceso de modernización tecnológica e institucional, ahora puede quedar rezagado en aspectos de expansión, respuesta social y cambio climático. Mientras en diversos contextos se ha logrado vincular a las comunidades, sacándolas del simple rol de consumidoras a ser pieza angular del proceso de generación, distribución y autosuficiencia energética, en nuestro país el peso de algunas entidades parece generar barreras de acceso para dinamizar el servicio.

Aunque se han configurado planes de desarrollo, estos siguen asociados y concentrados en proyectos tradicionales, los cuales incluso empiezan a ser percibidos por la industria como insuficientes para sus necesidades. Del mismo modo, la manera como se viene enfrentando el cambio climático demanda una actuación y el despliegue de una política decidida tanto por parte del Gobierno nacional como de los gobiernos locales. Finalmente, frente a la evolución 
del sector, se requieren estudios que contrasten las necesidades, las acciones realizadas y los procesos de responsabilidad que pueden asumir quienes participan en él.

\section{Referencias}

Asociación Colombiana de Generadores de Energía Eléctrica (ACOLGEN). (2016). Soluciones Estructurales del mercado de gas para lograr mayor competitividad y sostenibilidad del sector. Recuperado de http://www. acolgen.org.co/

Ávila, C. y Hurtado, L. (2016). Evolución Sectorial de la Distribución y Comercialización de Energía Eléctrica en Colombia 2010-2013. Bogotá.

Barrera, F. y García, A. (2010). Desempeño del mercado eléctrico colombiano en épocas de niño: Lecciones del 2009-10. Un informe para la Asociación Colombiana de Generadores de Energía Eléctrica (Acolgen). Madrid: Acolgen. Recuperado de http://www.acolgen.org.co/wp-content/uploads/ documentos/ACOLGEN_DESEMPEN\%CC\%83O\%20DEL\%20 MERCADO\%20ELE\%CC \%81CTRICO\%20COLOMBIANO $\% 20$ E N \% 20 E P O C A S \% 20 D E \% 20 E L \% 20 N IN \% C C \% 83 O LECCIONES\%20DEL\%202009-2010.pdf

Comisión de Regulación de Energía y Gas (CREG). (2016). Resolución 029 de 2016. Por la cual se define un esquema de tarifas diferenciales para establecer los costos de prestación del servicio de energía eléctrica a usuarios regulados en el SIN para promover el ahorro voluntario de energía. Recuperado de http://apolo.creg.gov.co/Publicac.nsf/1c09d$18 \mathrm{~d} 2 \mathrm{~d} 5 \mathrm{ffb} 5 \mathrm{~b} 05256 \mathrm{eee} 00709 \mathrm{c} 02 / 11 \mathrm{~d} 21 \quad$ 8ae3d892c1605257f70004ed535?OpenDocument

Comisión de Regulación de Energía y Gas (CREG). (2016). Resolución 051 de 2016. Por la cual se finaliza la aplicación del esquema de tarifas diferenciales adoptado mediante la Resolución CREG 029 de 2016.

Congreso de la República de Colombia. (1994). Ley 142 de 1994. Servicios públicos domiciliarios. "Por la cual se establece el régimen de los servicios públicos domiciliarios y se dictan otras disposiciones". Diario Oficial, 41.433.

Congreso de la República de Colombia. (1994). Ley 143 de 1994. Por la cual se establece el régimen de generación, interconexión, transmisión, distribución y comercialización de electricidad en el territorio nacional, se conceden unas autorizaciones y se dictan otras disposiciones en materia energética. Diario Oficial, 41.434. 


\section{Contexto Loon-aime, F}

Contaduría General de la Nación. (2013). Resolución 743 de 2013. Por la cual se incorpora en el Régimen de Contabilidad Pública, el marco normativo aplicable para algunas empresas sujetas a su ámbito y se dictan otras disposiciones.

García, M. (Ed.). (2006). Sistemas de seguimiento a mercados eléctricos internacionales: aplicación a los países de la región andina. Bogotá. Universidad Nacional de Colombia.

Intercolombia S. A. (2016). Informe de gestión 2015. Recuperado de http:// www.intercolombia.com/

Interconexión Eléctrica S. A. E.S.P. (2016). Reporte Integrado de Gestión 2015. Recuperado de http://www.isa.co/

Isagen S. A. E.S.P. (2016). Informe de gestión 2015. Recuperado de https:// www.isagen.com.co/

Ministerio de Minas y Energía de Colombia (Minminas). (2009). Sector Energía Eléctrica (Parte 1). En Memorias al Congreso de la República 2008-2009 (pp. 132-171) Recuperado de https://www.minminas.gov.co/

Organización Latinoamericana de Energía (OLADE). (2013). Modelos de mercado, regulación económica y tarifas del sector eléctrico en América Latina y el Caribe-Colombia. Recuperado de https://www.olade.org/

Servicio Nacional de Aprendizaje (SENA). (2013). Caracterización del Sector Eléctrico Colombiano. Recuperado de http://www.cidet.org.co/

Sistema de Información Eléctrico Colombiano (SIEL). (2017). Información pública del sector eléctrico. Recuperado de http://www.siel.gov.co/

Superintendencia de Industria y Comercio (SIC). (2011). Estudios de Mercado. Análisis descriptivo y estructural del sector de Energía en Colombia. Recuperado de http://www.sic.gov.co/

Superintendencia de Servicios Públicos Domiciliarios. (2014). Régimen básico de los servicios públicos domiciliarios. Recuperado de https://www. superservicios.gov.co/normativa

Transelca S. A. E.S.P. (2016). Informe de gestión 2015. Recuperado de http:// www.transelca.com.co/

Unidad de Planeación Minero Energética (UPME). (2013). Plan de expansión de referencia. Generación - Transmisión 2013-2027. Bogotá: Ministerio de Minas y Energía. 
Unidad de Planeación Minero Energética (UPME). (2014). Plan indicativo de expansión de cobertura de energía eléctrica 2013-2017. Bogotá: Ministerio de Minas y Energía.

Unidad de Planeación Minero Energética (UPME). (2015). Plan energético nacional Colombia: ideario energético 2050 en Discusión. Recuperado de http://wwwl.upme.gov.co/Paginas/Plan-Energetico-Nacional-Ideario-2050.aspx

Unidad de Planeación Minero Energética UPME. (2015). Boletín estadístico de minas y energía 2010-2015. Recuperado de http://www1.upme.gov. $\mathrm{co} /$ PromocionSector/SeccionesInteres/Documents/Boletines/Boletin Estadistico_2010_2015.pdf

Unidad de Planeación Minero Energética (UPME). (2015). Plan de expansión de referencia. Generación - Transmisión 2015-2029. Recuperado de http://www1.upme.gov.co/Energia_electrica/Planes-expansion/PlanExpansion-2015-2029/Plan_GT_2015-2029_VF_22-12-2015.pdf

Unidad de Planeación Minero Energética(UPME).(2016). Proyección deprecios de los energéticos para generación eléctrica enero 2016 - diciembre 2035. Recuperado de http://www1.upme.gov.co/Hidrocarburos/publicaciones/ Proyeccion_de_los_precios_de_los_combustibles_junio_2016.pdf

Unidad de Planeación Minero Energética (UPME). (2016). Boletín estadístico de Minas y Energía 2012-2016. Recuperado de http://www1.upme.gov. co/Paginas/Noticias/BOLETIN-ESTAD\%C3\%8DSTICO-DE-MINASY-ENERG\%C3\%8DA-2012---2016.aspx

\section{Para citar este artículo:}

León-Paime, F. (2019). Desafíos contemporáneos en el desarrollo del sector eléctrico colombiano. En-Contexto, 7(11), 87-111. 